\title{
O PROTOCOLO DE MANCHESTER NO SISTEMA ÚNICO DE SAÚDE E A ATUAÇÃO DO ENFERMEIRO
}

\author{
Valdeci de Assis TEIXEIRA ${ }^{1}$ \\ Gleidson Brandão OSELAME ${ }^{2}$ \\ Eduardo Borba NEVES ${ }^{3}$
}

\begin{abstract}
${ }^{1}$ Enfermeiro. Centro Universitário Campos de Andrade. Curitiba PR. Rua Irmã Sophia Rieche, $\mathrm{n}^{\mathrm{o}} 300$ - Cidade Industrial - Curitiba - PR. Telefone: 04184361817 - e-mail: assisesilvana@ yahoo.com.br

${ }^{2}$ Enfermeiro. Especialista em Saúde Pública. Docente do Centro Universitário Campos de Andrade. Curitiba PR. Rua João Scuissiato, $\mathrm{n}^{\circ} 1$ - Santa Quitéria. Telefone 04188741267 - e-mail: gleidsonoselame@ @mail.com

${ }^{3}$ Fisioterapeuta. Doutor em Saúde Pública e Meio Ambiente. Docente do Centro Universitário Campos de Andrade. Curitiba - PR. Rua João Scuissiato, n 1 - Santa Quitéria - e-mail: borbaneves@ hotmail.com
\end{abstract}

Recebido em: 27/09/2014 - Aprovado em: 27/11/2014 - Disponibilizado em: 15/12/2014

RESUMO: Objetivou-se analisar os princípios e motivos que levaram à implantação do Protocolo de Manchester nos hospitais públicos que integram o Sistema Único de Saúde. Realizou-se um estudo de caráter bibliográfico, por meio da busca eletrônica nas bases Scientific Eletronic Library Online (Scielo), Biblioteca Virtual em Saúde (Bireme) e Google Acadêmico, no período compreendido entre setembro de 2011 a março de 2012. O critério de inclusão foi de corte cronológico, utilizando-se artigos publicados nos últimos 08 anos. Optou-se pela técnica de análise de conteúdo, resultando em cinco categorias temáticas. Observa-se uma queda considerável nos índices de internamentos com consequente diminuição das taxas de mortalidade após implantação do protocolo de Manchester em hospitais públicos. No Brasil, o protocolo tem se mostrado eficiente e de fácil aplicação, além de possibilitar ao Estado o acompanhamento e auditoria relativos ao mesmo. Nesse processo, o Enfermeiro se apresenta como profissional qualificado para atuar como classificador do protocolo, e sua principal responsabilidade é a de se tornar proativo na avaliação rápida e eficiente dos casos de urgência e emergência, desde que salvaguardados os direitos do usuário.

Palavras-chave: Protocolo de Manchester; Sistema único de saúde; Enfermagem; Urgência e emergência; Triagem.

ABSTRACT: Was aimed to analyze the principles and reasons that led to the implementation of the Protocol Manchester in public hospitals that make up the Health System conducted a study of bibliographical character, through electronic searching databases Scientific Electronic Library Online (scielo), Virtual Health Library (BIREME) and Google Scholar, in the period September 2011 to March 2012 the inclusion criterion was chronological cut, using articles published in the last 08 years. We opted for the technique of content analysis, resulting in five thematic categories. There has been a considerable drop in the rates of hospitalizations with a consequent decrease in mortality rates after implementation of the protocol of Manchester in public hospitals. In Brazil, the protocol is efficient and easy to use, and enable the State to monitor and audit for the same. In this process, the nurse introduces himself as qualified to act as classifier of professional protocol, and its main responsibility is to become proactive in fast and efficient evaluation of urgent and emergency cases, since safeguarding the rights of the user.

Keywords: Manchester Protocol; Public health system; nursing; Emergency care; Screening.

\section{INTRODUÇÃO}

Instituído pela Constituição

Brasileira de 1988 e regulamentado pelas

Leis $8080 / 90$ e $8142 / 90$, o Sistema Único de Saúde (SUS) foi criado a fim de garantir

o acesso livre e gratuito a todos que necessitam de cuidados de saúde, cabendo ao Estado promover, através de políticas 
públicas, a diminuição das doenças e outros agravantes que coloquem em risco a saúde dos cidadãos. Para tanto, deve garantir a prevenção e proteção nas ações e nos serviços de saúde, e que o acesso a ele seja universal e igualitário ${ }^{(1,2)}$.

No âmbito do SUS, a instituição hospitalar pública ou a ela conveniada se configura como ferramenta com a incumbência de proporcionar assistência e tratamento a todos os usuários que a ela recorrem e que apresentem agravos de saúde. Com funcionamento ininterrupto durante as 24 horas diárias, os serviços de urgência e emergência configuram-se como portas de entrada na maioria desses hospitais $^{(3)}$.

Nas últimas décadas, a crescente procura por atendimentos de urgência e emergência nos hospitais públicos do país tem resultado em superlotação, o que contribui para provocar a uma desorganização do processo de trabalho, tanto do setor quanto dos profissionais que atuam nesses serviços, tendo como consequência a dificuldade nos atendimentos e nas internações ${ }^{(4)}$.

O Ministério da Saúde, em parceria com as Secretarias Estaduais e Municipais de Saúde, e buscando solucionar as reais necessidades dos usuários do sistema, implementaram políticas públicas no intuito de reorganizar a assistência prestada pelo SUS. Assim, foram adotadas diversas medidas, e entre elas destaca-se a implantação do acolhimento com classificação de risco, tendo como principal objetivo a organização do fluxo de usuários ${ }^{(5)}$.

Nessa perspectiva, o protocolo de avaliação e classificação de risco, fundamentado em experiências internacionais, constitui-se como ferramenta necessária e de grande utilidade nos serviços de urgência e emergência, estabelecendo o atendimento não mais pela ordem de chegada, mas sim pela gravidade do quadro e/ou queixa apresentada pelo paciente $^{(6)}$. 
O sistema de triagem de

Manchester originou-se em 1997 na cidade de Manchester, na Inglaterra $^{(7)}$, ante a necessidade do aprimoramento na qualidade da assistência nos serviços de urgência e emergência, a partir da priorização dos pacientes em virtude ao risco apresentado, reorganizando o fluxo nesses atendimentos ${ }^{(8)}$. Sua implantação ocorreu progressivamente em todos os hospitais do Reino Unido, sendo adotado por diversos países da Europa ${ }^{(9)}$.

Dentro deste contexto, o enfermeiro vem se mostrando como o profissional de saúde mais indicado para avaliar e classificar os riscos apresentados pelos pacientes que chegam aos prontos-socorros dos hospitais públicos. Suas ações são orientadas por protocolos que avaliam o nível da gravidade, determinando prioridades de atendimento e área de tratamento, o que configura uma assistência com segurança e competência ${ }^{(10)}$.
Considera-se o protocolo de Manchester uma ferramenta sensível na identificação de prioridades em setores de emergência. Baseia-se em um sistema de algoritmos composto por cinco níveis de urgência associados a cores, determinando tempo estimado e prioridade para o atendimento. No Brasil, este tipo de sistema foi adotado inicialmente pelo estado de Minas Gerais a partir do ano de $2007^{(11)}$.

A implantação de um sistema de classificação de risco, no caso, o Protocolo de Manchester, encontra por vezes uma espécie de resistência dadas as dificuldades relacionadas à introdução da nova tecnologia, e que é totalmente informatizada. Na falta de mais e melhores recursos públicos de pessoal e tecnologia, a implementação do Protocolo de Manchester torna difícil para muitos profissionais vincular essa prática à sua rotina diária, tornando a classificação dos pacientes em um processo longo e árduo $^{(12)}$. Sugere-se que tal fato decorre 
muito mais do que simplesmente adaptação

à rotina profissional, entende-se que fatores como carga de trabalho e falta de condições adequadas podem contribuir para este tipo de comportamento.

Diante disto, o presente estudo teve como objetivo analisar os princípios e motivos que levaram à implantação do Protocolo de Manchester nos hospitais públicos ligados ao o SUS, descrevendo a metodologia utilizada, como também o impacto provocado nos profissionais de saúde frente a essa nova tecnologia.

\section{MÉTODO}

Realizou-se um estudo de caráter bibliográfico, aquele que explica um tema ou problema com base em referências teóricas já elaboradas e publicadas, na busca de analisar e compreender outras pesquisas científicas existentes ${ }^{(13)}$.

A busca ocorreu no período compreendido entre setembro de 2011 à março de 2012, tendo como critérios de inclusão artigos publicados nos últimos 08 anos, por entender que com um corte cronológico mais prolongado incorreria no risco de confrontar, em um mesmo estudo, publicações com técnicas diferentes das atuais, dado que o Protocolo de Manchester começou a ser implantado com mais vigor a partir do ano de 2007 no Brasil.

Os dados foram obtidos com busca eletrônica nas bases Scientific Eletronic Library Online (Scielo), Biblioteca Virtual em Saúde (Bireme) e Google Acadêmico, através da utilização dos termos “Acolhimento com Classificação de Risco", "Protocolo de Manchester" e "Resistência dos Enfermeiros". Para análise do material, os termos "triagem" e “classificação de risco" foram considerados como sinônimos.

A categorização dos dados deu-se pela técnica de análise de conteúdo, que implica em classificar os elementos constitutivos de um conjunto por diferenciação e, em seguida, por reagrupamento em categorias, segundo a 
analogia entre os elementos encontrados. As categorias, portanto, são rubricas ou classes que reúnem um grupo de elementos com caracteres comuns sob um título genérico $^{(13)}$.

\section{RESULTADOS}

Foram encontrados 26 trabalhos, excluindo-se as duplicações entre as bases de dados pesquisadas. Destes, apenas 10 apresentaram conteúdo de interesse ao objetivo deste estudo. Esses 10 trabalhos tiveram seu conteúdo analisado e foi possível identificar cinco categorias temáticas: "a superlotação nos serviços de urgência e emergência nos hospitais ligados ao SUS”, “a adoção da classificação de risco no SUS como processo de triagem”, “ações governamentais para a implantação do sistema de classificação de risco", "sistema de triagem de Manchester e sua aplicabilidade", e "a capacitação do Enfermeiro para o uso do protocolo de Manchester".

\section{DICUSSÃO}

\section{A superlotação nos serviços de urgência \\ e emergência nos hospitais ligados ao SUS}

Os serviços de urgência e emergência constituem-se num importante componente da assistência à saúde para os doentes que procuram por atendimento nos hospitais públicos do SUS. Nas últimas décadas, observou-se uma elevada e constante procura de cuidados nesses serviços ${ }^{(4)}$. Isso se deve a fatores como o aumento da expectativa de vida da população, acarretando a incidência das doenças crônicas e degenerativas, somadas a fatores de risco como o tabagismo, obesidade, sedentarismo, abuso do álcool e outras drogas, além da alimentação inadequada ${ }^{(14)}$.

A constante e crescente procura por estes serviços pode ser associada ao aumento da violência urbana, dos acidentes de trânsito e mesmo pela criação e implantação de novas tecnologias. Graças 
a estas últimas, a população tem tido mais facilidade de acesso a fontes de informação - tanto técnicas como políticas -, capacitando-a a exigir mais de todos os serviços nos quais tem direito ao atendimento, o que viria, no limite, a colocar em risco a viabilidade do SUS, obrigando os gestores (âmbitos federal, estadual e municipal) a uma reengenharia na organização e no modelo do atendimento $^{(15)}$.

Também alguns serviços de atenção básica são responsáveis pelo referido crescimento ao encaminhar pacientes que não apresentam risco compatível com urgência/emergência e que deveriam ser atendidos naquelas mesmas unidades. Como resultado, nota-se um congestionamento nos prontos-socorros dificultando a adequada prestação de serviços, o que gera insatisfação de todos os usuários e profissionais de saúde ${ }^{(16)}$.

Por sua vez, a falta de capacitação organizacional do setor público hospitalar contribuiu para o aumento expressivo da carga de trabalho nos serviços de urgência e emergência. Uma consequiência imediata é a sobrecarga de doentes nesse setor, provocando um desgaste excessivo dos profissionais de saúde ${ }^{(17)}$, dificuldades no atendimento, recusa de pacientes oriundos de outras unidades, culminando num atendimento fragmentado e a um total desarranjo nas portas do $\operatorname{SUS}^{(4)}$.

\section{A adoção da classificação de risco no}

\section{SUS como processo de triagem}

Os fundamentos éticos e constitucionais levam à afirmação de que o acesso aos serviços de saúde é um direito inerente a todos os brasileiros ${ }^{(18)}$. No entanto, fundamentalmente por questões econômicas, questiona-se até que ponto esse direito é praticável, pois as necessidades de saúde tornaram-se maiores do que os recursos disponibilizados pelo Estado para esse fim. Como tais custos vão além das disponibilidades para gastos, impõe-se a necessidade de se estabelecer $\operatorname{prioridades}^{(19)}$. 
Diversos países passaram pelo

mesmo problema e encontraram no processo de triagem parte da solução, ao estabelecer processos de avalição clínica rápidos, a fim de determinar o tempo e o setor em que os pacientes deveriam receber atendimento em uma unidade de urgência e emergência com recursos $\operatorname{limitados}^{(19)}$. Devido ao sucesso, um desses processos, o Protocolo de Manchester, o levou a ser copiado em várias partes do mundo ${ }^{(20)}$. Neste contexto, a triagem de prioridades proporciona ao paciente uma melhor avaliação do seu estado de saúde ${ }^{(21)}$. Desta forma, os serviços de triagem tornaram-se essenciais ao SUS, à necessidade de organizar o fluxo e selecionar os meios adequados para o diagnóstico e tratamento $^{(7)}$.

Apesar de o termo triagem ser consagrado mundialmente, sua utilização não é universal. Na Espanha, por exemplo, seu uso se limita a situações catastróficas $^{(22)}$. Em Santa Catarina na catástrofe climática de 2008, por exemplo, confundia-se com o ato de "mandar o paciente embora, sem atendimento". Para evitar esse transtorno ou criar algum tipo de resistência, adotou-se o termo classificação de risco como sinônimo de triagem. Entretanto, apesar da recomendação do Ministério da Saúde, diversos profissionais e autores ainda continuam a utilizar o termo triagem ${ }^{(10)}$.

\section{Ações governamentais para a} implantação do sistema de classificação de risco

No intuito de reorganizar o setor, o Ministério da Saúde, por meio da Portaria 2.048/2002, determinou princípios e diretrizes a serem seguidos nos sistemas de urgência e emergência do país, estabelecendo normas e critérios para operação e prestação desses serviços, cadastrando e classificando os hospitais públicos que prestam esse tipo de atendimento, além da determinar a criação de coordenações em nível estadual ${ }^{(6)}$. 
O objetivo maior da Portaria

2.48/2002 foi estruturar o sistema de saúde de tal forma que toda a rede estivesse interligada, envolvendo desde o atendimento pré-hospitalar até o de alta complexidade, capacitando cada elemento envolvido na prestação da assistência e responsabilizando-o pela atenção prestada dentro dos limites da sua qualificação ${ }^{(6)}$.

No entanto, em 2004, os problemas com a superlotação ainda persistiam. O Ministério da Saúde lança, então, a Política Nacional de Humanização $(\mathrm{PNH})^{(23)}$, e dentro desta propõe a implantação do Acolhimento com Classificação de Risco (ACR) como forma de criar condições para que todos os usuários que procurassem por esses serviços fossem atendidos ${ }^{(24)}$. A ACR permite identificar os pacientes que necessitam de cuidados imediatos, a partir do potencial de risco e/ou grau de sofrimento apresentados por aqueles que apresentam maior grau de gravidade clínica são priorizados em detrimento àqueles que apresentam quadros clínicos menos graves e que podem aguardar pelo atendimento por um período maior de tempo ou serem encaminhados a unidades de pronto atendimento. Em outros termos, o ACR proporciona agilidade no atendimento e organiza o fluxo no setor ${ }^{(4)}$.

\section{Sistema de triagem de Manchester e sua aplicabilidade}

No Brasil, o estado de Minas Gerais foi o primeiro a utilizar o Protocolo de Manchester nos postos de atenção à saúde. Para sua implantação foi necessária uma negociação envolvendo o governo mineiro com o Grupo Português de Triagem (GPT), possuidor dos direitos para tradução e utilização desse protocolo, sob autorização do British Medical Journal (BMJ) e a Manchester Triage Group (MTG), detentores da propriedade dos direitos autorais. Para a implantação foi também necessária a criação do Grupo Brasileiro de Classificação de Risco (GBCR), o qual firmou acordo com o GPT, MTG e o BMJ e obteve autorização para 
representar e utilizar o protocolo no País (25).

Curitiba foi a $2^{\mathrm{a}}$ cidade do Brasil a implantar o Protocolo de Manchester em sua rede de unidades de saúde. Em 24 de maio de 2011, no Centro Municipal de Urgências Médicas (CEMUM), na Regional do Sítio Cercado, a classificação de risco foi implantada em fase experimental, logo sendo estendida às 109 unidades de atenção básica, aos outros 07 CEMUM e as unidade de Saúde da Família $^{(26)}$.

Fundamentado por conceitos internacionais, o Sistema de Triagem de Manchester impõe a padronização no atendimento das emergências ao estabelecer a necessidade de preferência ao atendimento a partir da indicação clínica. Esta busca de minimizar os problemas encontrados ${ }^{(27)}$, tendo como principal objetivo o estabelecimento de prioridades, com base em critérios de gravidade sistemáticos, onde se leva em conta o problema do paciente, a história clínica, os sinais e sintomas apresentados, os sinais vitais e a condição física, permitindo definir a prioridade clínica a um tempoalvo recomendado durante o aguardo do atendimento médico ${ }^{(28)}$.

O Protocolo de Manchester é composto por 52 fluxogramas que refletem as diversas condições relacionadas à classificação de risco, sendo acompanhado de nota explicativa definindo sua aplicabilidade, facilitando e orientando o profissional sobre o direcionamento da situação para o fluxograma mais adequado $^{(29)}$. Essa classificação é realizada quando o paciente chega às portas do sistema, a fim de que doentes menos graves não sejam priorizados em detrimento àqueles que apresentam quadros verdadeiramente graves ${ }^{(7)}$.

\section{Capacitação do Enfermeiro para o uso}

\section{do Protocolo de Manchester}

A Portaria 2048/2002 estabelece que as unidades de urgência e emergência, na realização do processo de acolhimento e 
classificação de risco, tenham um profissional de saúde com formação superior, exigindo como critério o seu treinamento específico na utilização de protocolos informatizados $^{(30)}$.

Atualmente, inúmeros hospitais públicos do país, que adotaram o sistema de triagem de Manchester, totalmente sistematizado, têm atribuído ao Enfermeiro a responsabilidade na avaliação e classificação de pacientes em concordância ao Protocolo, determinando suas necessidades e prioridades $^{(14)}$. Essa atribuição se deve ao fato de que durante sua vida profissional - dado o peculiar processo de trabalho - o Enfermeiro pode adquirir habilidades fundamentadas por uma escuta qualificada, observação e registros detalhados, capacidade de trabalho em equipe, tomada de decisão a partir de um raciocínio clínico e rápido, identificando nos pacientes tanto as necessidades biológicas como também as psicológicas e sociais $^{(10)}$.
Os Conselhos Regionais de Enfermagem (COREN) de alguns estados têm emitido pareceres em referência à atuação do Enfermeiro no processo da classificação de risco. Entre eles, citamos o Parecer 010/2007, do COREN de Minas Gerais, ressaltando que o Enfermeiro deve ser devidamente capacitado para proceder a classificação de risco, e estando subsidiado por protocolo técnico ${ }^{(12)}$. O Parecer 0064/2011, do COREN do estado do Tocantins considera o Enfermeiro como o profissional detentor de conhecimentos, capacitado para avaliação de pacientes, direcionado por um protocolo e/ou portaria de classificação de atendimento, conforme o estado geral apresentado pelo paciente ou consulta de enfermagem ${ }^{(31)}$.

O estudo constatou que a implantação do sistema de classificação de risco do Protocolo de Manchester tem encontrado uma inevitável resistência por parte de muitos Enfermeiros que, devido ao longo tempo do exercício profissional em prontos-socorros de diversos hospitais, 
desenvolveram uma habilidade própria de avaliar e identificar as reais necessidades dos pacientes. Habilidosos no preenchimento e registro detalhado em todo o tipo de formulário e/ou documento, o que os leva a se autointitularem de “experientes", eles estranham a implementação de novos conceitos que resultam em mudanças de paradigmas, opondo-se ao surgimento e/ou implantação das novas tecnologias ${ }^{(32)}$. No setor saúde, a tecnologia ainda é marcada pela conformação entre saúde e doença, normal e patológico, vida e morte, tal fato em que os profissionais não podem intervir, o que leva a crer que o conhecimento é o maior patrimônio tecnológico de atenção a saúde. Desta forma, a tecnologia é tida muitas vezes como concorrente ${ }^{(33)}$.

Essa resistência se dá pela exigente adaptação relacionada à introdução da nova tecnologia - o Protocolo de Manchester - que vem totalmente informatizada. Para muitos profissionais, vincular essa prática à sua rotina diária é um arranjo difícil, o que torna a classificação dos pacientes em um processo longo e árduo. No entanto, essa mudança deve ser vista pelos profissionais como um aperfeiçoamento do sistema de triagem proporcionando o desenvolvimento de um raciocínio incisivo na tomada da decisão, bem como o aprimoramento das competências do Enfermeiro na atenção em urgência e emergência ${ }^{(20)}$.

\section{CONSIDERAÇÕES FINAIS}

A superlotação dos serviços de saúde aliada à fragmentação do processo de trabalho culminaram no desrespeito aos direitos humanos, fato que deve levar o profissional da saúde a uma reflexão no intuito de buscar formas de como superar tal impasse. Isto requer a adoção de uma atitude que possa ser compreendida e introduzida no sistema com o objetivo único de prestar uma assistência com qualidade, baseada num modelo de atenção resolutivo, e realizada de forma 
humanizada e acolhedora, resultando em satisfação por parte dos usuários.

Neste sentido, diversas publicações atestam uma queda considerável nos índices de internamentos, com consequente diminuição das taxas de mortalidade, após a implantação do sistema de classificação de risco trazida pelo Protocolo de Manchester. No Brasil, a implementação do Protocolo tem se mostrado eficiente seja em qualquer nível de atendimento, além de possibilitar ao Estado o acompanhamento, em tempo real, de informações quanto ao local e momento dos casos assistidos. Sendo o Enfermeiro entendido como profissional qualificado a atuar como classificador do Protocolo, ou seja, sua principal atuação tem sido a prestação de uma avaliação rápida e eficiente dos casos de urgência/emergência. Ele tem ciência do perfil e da gravidade dos quadros apresentados, além de conhecimento do processo de trabalho da equipe de profissionais envolvidos, podendo haver a delegação de tarefas.

Avalia-se que, com a inclusão dessa nova tecnologia de classificação de risco, a habilidade do profissional será cada vez mais requisitada e, por extensão, aprimorada a sua competência na operação de equipamentos informatizados que funcionam como suporte decisivo na classificação dos doentes de urgência/emergência. $\mathrm{O}$ fato da resistência pode ser encarado como temporário, visto os benefícios trazidos pelo protocolo nos diversos locais onde já foi implantado. Comumente o medo do novo é apresentado na forma de resistência, porém, em alguns casos pode ser entendido como despreparo.

Desta forma, a contínua capacitação dos enfermeiros torna-se fundamental, exigindo atualização constante relacionada à realidade vivenciada no ambiente de trabalho, e com o desenvolvimento de uma visão critica que leve o profissional a refletir sobre o seu modo de trabalhar, sem descuidar-se da arte do cuidado. 


\section{REFERÊNCIAS}

1. Brasil. Constituição da República Federativa do Brasil. Brasília: Senado.1988.

2. Ministério da Saúde (BR). Lei Orgânica da Saúde n. 8.080 de 19 de setembro de 1990. Brasília: Ministério da Saúde; 1990.

3. Nicácio F, Campos GWS. Instituições de "portas abertas": novas relações usuários-equipes-contextos na atenção em saúde mental de base comunitária/territorial. Rev. Ter. Ocup. Univ. 2005; 16(1): 40-46.

4. Albino RM, Grosseman S, Riggenbach V. Classificação de risco: uma necessidade inadiável em um serviço de emergência. ACM arq catarin Med. 2007; 36(4): 70-75.

5. Ministério da Saúde (BR). Secretaria Executiva, Núcleo Técnico da Política Nacional de Humanização. HumanizaSUS - Acolhimento com classificação de risco: um paradigma ético no fazer em saúde. Brasília: 2004.

6. Ministério da Saúde (BR). Portaria $\mathrm{n}^{\circ}$ 2.048, de 5 de novembro de 2002. Dispõe sobre o regulamento técnico das urgências e emergências e sobre os serviços de atendimento móvel de urgências e seus diversos veículos de intervenção. Brasília: 2002.

7. Hay E, Bekerman L, Rosemberg G. Peled R. Quality assurance of nurse triage: consistency of results over three years. Am J Emerg Med 2001; 19(2):113-17.

8. Scharam FR. Da bioética privada à bioética pública. In: Fleury $\mathrm{S}$, organizadora. Saúde e democracia: a luta do C.E.B.E.S., São Paulo: Lemos Editorial; 1997. P 227-40.

9. Quilete JB, Oliveira ALG, Xavier BLS, Gomes SR. Acolhimento com classificação de risco no serviço de emergência: tecnologia de cuidado que assegura acesso universal, resolutivo e humanizado no atendimento. In: IX Congresso Brasileiro de Saúde Coletiva, 2009; Recife, Brasil. Associação Brasileira de Pós-Graduação em Saúde Coletiva/ABRASCO; 2009.

10. Souza CC. Grau de concordância de classificação de risco de usuários atendidos 
em um pronto-socorro utilizando dois

diferentes protocolos [dissertação].

Universidade Federal de Minas Gerais.

Belo Horizonte (MG); 2009.

11. Souza CC, Toledo AD, Tadeu LFR,

Chianca TCM. Classificação de risco em

pronto-socorro: concordância entre um

protocolo institucional Brasileiro e

Manchester. Rev. Latino-Am.

Enfermagem. 2011; 19(1): 26-33.

12. Conselho Regional de Enfermagem de Minas Gerais. Parecer Técnico $n^{\circ} 10$, de 22 de fevereiro de 2007. Dispõe sobre a participação do enfermeiro na triagem de pacientes sem a presença de médicos especialistas. Belo Horizonte (MG): Conselho Regional de Enfermagem de Minas Gerais; 2007.

13. Gatti MFZ. Triagem de enfermagem em serviço de emergência. Centro de Estudos e Pesquisa. Boletim Científico do Hospital Samaritano. São Paulo; 2003. 14. Machado SCEP, organizadores. Como um protocolo de classificação de risco pode qualificar o encaminhamento dos pacientes na emergência. Ministério da Educação. Hospital de Clínicas de Porto Alegre (RS); 2005.

15. Gil AC. Como elaborar projetos de pesquisa. São Paulo: Atlas; 2002.

16. Fontes P. Reflexão bioética sobre a priorização e o racionamento dos cuidados de saúde: entre a utilidade social e a equidade. Cad. Saúde Pública. 2008; 24(3): 696-701.

17. Ministério da Saúde (BR). Anuário Estatístico de Saúde do Brasil 2001. Brasília; 2001.

18. Marques AJS, Mendes Junior EV, Silva OA, Silva MVCP. O choque na gestão da saúde em Minas Gerais. Belo Horizonte: Secretaria do Estado de Saúde. 2009. p. $81-84$.

19. Ministério da Saúde (BR). Política Nacional de Atenção às Urgências. Brasília (DF): Ministério da Saúde; 2006.

20. Melo MCB, Vasconcellos MC, organizadores. Manual de atenção às urgências e emergências em pediatria. Belo Horizonte: Escola de Saúde Pública 
de Minas Gerais, 2005.

21. Robertson-Steel I. Evolution of triage systems. Emergency Medical Journal. 2006; 23(1):154-155.

22. Sánchez FJM, Castilho JGD, Zamorano J, Candel FJ, Armengol JJG, Villarroel P, Elvira C, Farré AL. The physician as a necessary participant in triage in the emergency department of a tertiary hospital. Emergências. 2008; 20(2): 41-47.

23. Romero N, Lozano J. De las funciones y tareas propias de los diplomados de enfermería en la recepción, acogida y clasificación de los pacientes en el área de urgencias. Recomendacion científica 99/01/01 de 15 de junio de 1999, de La Sociedad Española de Enfermería de Urgencias (SEEU): 2006.

24. Ministério da Saúde (BR). HumanizaSUS - Política Nacional de Humanização: a humanização como eixo norteador das práticas de atenção e gestão em todas as instâncias do SUS. Brasília: Ministério da Saúde, 2004.
25. Grupo Português de Triagem. Triagem no serviço de urgência. Manual do formando. Lisboa: BMJ Publishing Group, 2002.

26. Centros de urgência de Curitiba terão classificação por cores. O Estado do Paraná [Internet] Paraná; 2011 [acesso em 25 mai 2012]. Disponível: www.paranaonline.com.br.

27. Mackway-Jones K, Marsden J, Windle J. Emergency triage / Manchester Triage Group. 2nd ed. Oxford: Blackwell Publishing, 2006.

28. Cronin J. The introduction of the Manchester triage scale to an emergency department in the Republic of Ireland. Accident and Emergency Nursing. 2003; 11(2): 121-125.

29. Freitas P. Triagem no serviço de urgência: grupo de triagem de Manchester. Portugal: BMJ Publishing Group, 2002.

30. Conselho Regional de Enfermagem do Tocantins. Parecer Técnico $\mathrm{n}^{\circ} 0064$. Dispõe sobre a triagem feita pela 
enfermagem no pronto-socorro. COREN-

TO; 2011.

31. Bernardo CG. A resistência a mudanças em uma organização: uma análise à luz do processo controle integrado de mudanças do protocolo de Manchester [dissertação]. Universidade de São Paulo, São Paulo, 2005.
32. Marques AJS, Mendes Junior EV, Silva OA, Silva MVCP. O choque na gestão da saúde em Minas Gerais. Belo Horizonte: Secretaria de Estado de Saúde de Minas Gerais, 2009.

33. Silva DCS, Alvim NAT, Figueiredo PA. As tecnologias leves em saúde e sua relação com o cuidado de enfermagem hospitalar. Esc. Anna Nery Rev Enferm. 2008; 12(2): 291-8. 